\title{
Introduction to Government Innovation in the Digital Age Minitrack
}

\author{
Muhammad Mustafa Kamal \\ Brunel University London \\ muhammad.kamal@brunel.ac.uk
}

\author{
J. Ramon Gil-Garcia \\ University at Albany - SUNY \\ jgil-garcia@ctg.albany.edu
}

\author{
Ulf Melin \\ Linköping University \\ ulf.melin@liu.se
}

Government innovation through the development, implementation and use of digital technologies is on every agenda globally. Both practice and research communities suggest that the use of information and communication technologies (ICT) in governments and societies at large has substantial innovation potential. Phenomena like the sharing economy, big or open data, automation, robotization, Internet of Things (IoT), social media and, more recently, blockchain technologies, just to mention a few, significantly impact government organizational structures, managerial and financial practices, and overall culture. ICT-enabled innovation in government agencies through emergent technologies could significantly change governments and their relationships with citizens, being driven both from inside a government organization, from politicians and decisions makers, or from external stakeholders such as citizens, individual practitioners or companies. From a research point of view, it is important to study innovative practices in government from different perspectives, and to critically and jointly examine technologies, policies, practices, and patterns of success and failure. When doing this, we can treat digital government as a multidisciplinary and multidimensional phenomenon in order to understand and to explain its potential for ICTenabled government transformation and processes of digitalization.

In this minitrack, we welcome studies focusing on different perspectives, levels or aspects of strategic, political, institutional, managerial, organizational, individual, legal, economic, and external relationship factors within digital government and, particularly, ICT-enabled government innovations. We welcome papers that focus on innovation and the transformational aspects of digital government as well as their implications for government(s) and the society as a whole. We also seek papers that clearly establish an understanding of how technology can enable governments and their leadership to improve government efficiency, making decision-making process more transparent, interacting with their citizenry and, most importantly, developing trust between governments and citizens. We invite empirical, theoretical, and/or conceptual contributions, with diverse research approaches and techniques that show the importance of governmental, institutional, organizational, managerial, and democratic aspects of innovation in digital government.

This minitrack acknowledges the complexity of governing, organizing, and managing digital government innovations and their transformative potential especially in the context of emerging phenomena using new digital technologies. Increasingly, this involves inter-organizational and cross-sector collaboration and co-creation of value, and cross-jurisdictional networks, and the management of a large variety of relationships with both internal and external stakeholders. Research Topics include, but are not limited to:

- Government innovation through digital technologies - conceptual and critical perspectives.

- $\quad$ Policies, strategies, culture and value foundations for ICT-enabled government innovation.

- Theoretical foundations and perspectives on digitalized government.

- The role of ICT, people and processes of digitalization government.

- Critical, success and failure focused studies of contemporary development and use of digital technology in governments.

- Strategies for the design, development, implementation, maintenance, performance, and evaluation of e-Government applications and processes.

- Multidisciplinary and multidimensional perspectives on digital government.

- Governance, accountability, and transparency in digital government.

- Service transformation, innovation and multichannel service delivery.

- Integration of government data (big, open etc.), services, and/or processes.

- Transformational aspects and implications of data analytics, policy informatics, and smart cities.

- Change management in e-Government initiatives focusing on continuous improvement and radical change/innovation. 
- Engagement, stakeholders and e-participation initiatives within digital government.

- Efficiency and/or effectiveness and the creation of value through e-Government initiatives.

- Stakeholder engagement in digital governments.

- Cross-government and cross-sector information sharing, regional, national and transnational information sharing networks, information integration, privacy, and security.
- Impact, outcomes, and implications of eGovernment initiatives and related methods and frameworks for evaluation.

- Political, economic, legal, and social aspects of emerging digital government.

- Organizational, managerial, and governance aspects of government transformation through digital technologies. 